\title{
Kinematics of early-type galaxies in compact groups ${ }^{\star, \star \star}$
}

\section{HCG 67, HCG 74, and HCG 79}

\author{
P. Bonfanti ${ }^{1}$, F. Simien ${ }^{2}$, R. Rampazzo ${ }^{1}$, and Ph. Prugniel ${ }^{2}$ \\ 1 Osservatorio Astronomico di Brera, Via Brera 28, I-20121, Milano, Italy \\ 2 CRAL-Observatoire de Lyon, CNRS UMR 142, F-69561 St-Genis-Laval Cedex, France
}

Received May 10; accepted August 3, 1999

\begin{abstract}
We present measurements of stellar kinematics for seven early-type galaxies in HCG 67, HCG 74, and HCG 79. These data are aimed at studying the relation between the environment and the dynamics, structure and stellar content of early-type galaxies. In the present three groups, the kinematic features we observed cannot be associated unambiguously to physical interactions. Visible morphological peculiarities do not appear correlated with kinematical perturbations.
\end{abstract}

Key words: galaxies: kinematic and dynamics; galaxies: interactions; galaxies: elliptical and lenticular, cD; galaxies: formation; galaxies: evolution

\section{Introduction}

The origin, evolution and even physical reality of Hickson Compact Groups (Hickson 1982) are still a matter of debate. Their morphology suggests that many individual HCG galaxies present signatures of interaction (see, e.g., Mendes de Oliveira \& Hickson 1994: hereafter MdOH). But it has been argued that a high fraction of HCGs are rather the result of chance alignments within loose groups (Mamon 1986, 1987, 1992, 1994) or within filaments (Hernquist et al. 1995; Ostriker et al. 1995). If studies on the evolution of HCGs have produced apparently conflicting conclusions (White et al. 1990; Hickson et al. 1992; Zepf 1993; Pildis 1995; Sulentic \& Rabaça

Send offprint requests to: F. Simien

e-mail: fs@obs.univ-lyon1.fr

* Based in part on observations obtained at the Observatoire de Haute-Provence.

** Table 3 and Table 4 are available from the CDS, Strasbourg, via anonymous ftp to cdsarc.ustrasbg.fr (130.79.128.5) or via http://cdsweb.ustrasbg.fr/Abstract.html
1994; Athanassoula et al. 1997), this probably reflects the wide diversity in the nature and evolution of these groups.

Studying early-type galaxies in such environment will be helpful to constrain the actual status of their host group, and also to characterize the reactions of early-type galaxies to gravitational perturbations. In this paper we focus on the kinematical aspects.

The presence of diffuse X-ray emission from the hot intra-group gas provides a strong evidence for the boundness of a system (Ponman et al. 1996, and references therein), but it is still difficult to ascertain the presence of such a diffuse background (dos Santos \& Mamon 1999). $\mathrm{H}$ I deficiency in many HCG spirals (Williams \& Rood 1987; Huchtmeier 1997), and concentration within their central regions (Menon 1995a,b) are other signatures of the existence of the intra-group medium. In addition, a group can be interacting but yet be unbound (and free of intra-group medium) if we observe it at an early stage of its evolution. In this case signatures of interaction can be searched for in an excess of star formation, from broadband colors, CO and FIR properties (Sulentic \& de Mello Rabaça 1993; Moles et al. 1994; Venugopal 1995; VerdesMontenegro et al. 1998; Leon et al. 1998); an alternative is to study the distribution of AGNs (de Carvalho \& Coziol 1999, and references therein). There is also the kinematics of spiral galaxies (Rubin et al. 1991: hereafter RFH; Mendes de Oliveira et al. 1997). In only a minority of cases it is possible to obtain clear evidence of interactions. For example, kinematical evidence have been found in HCG 16 (Mendes de Oliveira et al. 1998), HCG 67 (Bettoni \& Buson 1999: hereafter BB99), HCG 90 (Plana et al. 1998), and HCG 96 (Verdes-Montenegro et al. 1997).

Because of the above ambiguities, we selected earlytype galaxies in HCGs regardless of their X-ray status or previously reported evidence for interaction.

The effects of interactions on early-type galaxies are perhaps more subtle than on spirals, and stellar kinematics have been less systematically studied. In addition to 
morphological signatures such as shells or ripples, distorted isophotes (Davoust \& Prugniel 1988), these galaxies are expected to show transient features in their kinematic profiles, as in pairs of galaxies (Bonfanti et al. 1995; Combes et al. 1995). But the difficulty of connecting particular kinematic characteristics to interactions has been emphasized by Rampazzo et al. (1998). There are, however, some exceptions: in HCG 90, for example (Longo et al. 1994), strong perturbations in the velocity patterns could be unequivocally linked to direct interactions within the group.

With the aim of increasing the small amount of available data in stellar kinematics, we have looked for kinematic signatures in the early-type galaxies of three Hickson groups; these are HCG 67 (a strong source of diffuse X-rays), HCG 74 (whose members are embedded in a common envelope), and HCG 79 (with a high density of galaxies). In Sect. 2, imaging and spectroscopic material is presented. Our results are detailed in Sect. 3, and summarized in Sect. 4.

\section{The data}

\subsection{Broad-band images}

HCG objects were imaged using the 1.20-m telescope of the Observatoire de Haute-Provence, with a Tektronix $1024 \times 1024$ CCD. Each field covers a square of 11 ' 7 side, with a pixel size of 0 ". 67 . Exposure times of $600 \mathrm{~s}$ were secured through the $V$ filter. The seeing conditions were mediocre, and the usage of our images has been limited to morphological considerations only.

We have also benefited from images of much better resolution, kindly communicated to us by D. Bettoni (for HCG 67), and by P. Hickson (for all three groups); hereafter, these $R$-band frames will be referred to as DBAsiago and PH-CFHT images, respectively.

\subsection{Spectroscopic observations}

Long-slit spectra have been obtained at the 1.93-m telescope of the Observatoire de Haute-Provence, equipped with the CARELEC spectrograph. Spectra have a dispersion of $66 \AA \mathrm{mm}^{-1}$ corresponding to $1.78 \AA \mathrm{px}^{-1}$ on a $512 \times 512$ Tektronix CCD, with pixels of $27 \mu \mathrm{m}$; the actual spectral resolution is $3.2 \AA \mathrm{FWHM}$. The wavelength range covered is $\approx 4800<\lambda<5750 \AA$. The scale perpendicular to the dispersion is 1 !' 1 , the slit width was 2 .' 1 . The exposure time and slit position angles of the different spectra are presented in Table 1. K0 III stars were also observed, as templates.

\subsection{Analysis of spectra}

We have followed closely the technique used in Simien \& Prugniel (1997a, 1997b, 1997c, 1998), whose main characteristics are summarized again, for convenience. Pre-reduction, cosmic-ray removal in the outer regions, wavelength calibration and sky subtraction were performed with ESO-MIDAS. Multiple spectra of the same object were co-added after applying a proper relative shift, i.e.: a) in $\lambda$, using the calibration spectra to monitor the mechanical flexures within the spectrograph, and b) perpendicularly to the dispersion, to match the nucleus position along the slit, down to a fraction of pixel.

The kinematical parameters are determined by a Fourier-Fitting technique similar to that described in Franx et al. (1989). This allows the simultaneous calculation of the rotation velocity $V(r)$ and the velocity dispersion $\sigma(r)$, as a function of the distance $r$ from the center.

The removal of the cosmic-ray hits in the central regions is performed on the galaxy spectrum within the same procedure; it is based on a three-sigma clipping of residuals between the galaxy spectrum and the properly shifted, convolved star spectrum. The $V$ and $\sigma$ parameters are actually determined in two successive steps, using first the raw galaxy spectrum, and then the spectrum with cosmic rays removed as explained. Adjacent lines are averaged to enhance the $\mathrm{S} / \mathrm{N}$ ratio of the spectrum in the outer regions. For a spectrum at radius $r$, a weight is assigned to the neighboring lines $(r \pm \delta r)$ entering the average; for this weight, a Gaussian fall-off as a function of $\delta r$ was chosen, with a FWHM width between 0 and 3.6 pixels ( 0 and $4^{\prime \prime} .1$ ).

Our determinations of the heliocentric recession velocities $V_{0, \text { hel }}$ and central velocity dispersions $\sigma_{0}$ are collected in Table 3 (also available in electronic form). The radialvelocity and velocity-dispersion profiles are displayed in Fig. 3 and Fig. 6, and proposed in electronic form in Table 4. Individual results are discussed in Sect. $3 . \mathrm{MdOH}$ presented the redshift survey of the entire Hickson's sample; our measurements of the systemic velocities have been compared with their measurements and, for the seven galaxies in common, the average difference is $\left\langle V_{\text {ours }}-\right.$ $\left.V_{\mathrm{MdOH}}\right\rangle=-24 \pm 99 \mathrm{~km} \mathrm{~s}^{-1}$, which is unsignificant.

\section{Individual notes and results}

In this section, we comment on each group, summing up relevant results from the literature, and discussing our own results in this context. Basic morphological characteristics are shown in Fig. 1, which presents isophote maps for the groups.

\section{1. $H C G 67$}

\subsubsection{Morphology}

According to Ribeiro et al. (1998) this group is a loose one with a compactness increasing inwards; their kinematic analysis, which identifies 14 objects at $V_{\text {hel }} \approx$ 
Table 1. Journal of spectroscopic observations

\begin{tabular}{rrrrrr}
\hline $\begin{array}{r}\text { member } \\
\text { ident. }\end{array}$ & $\begin{array}{r}\alpha \\
(2000)\end{array}$ & $\begin{array}{r}\delta \\
(2000)\end{array}$ & $\begin{array}{r}\text { exp. time } \\
\text { (seconds) }\end{array}$ & $\begin{array}{r}\text { slit PA } \\
\text { (degrees NE) }\end{array}$ & $\begin{array}{r}\text { seeing FWHM } \\
\text { (arcsec) }\end{array}$ \\
\hline HCG 67a & 134911.3 & -71327.6 & $3 \times 3000$ & 37 & 2.7 \\
HCG 67d & 134909.7 & -71354.3 & $3 \times 3000$ & 37 & 2.7 \\
HCG 74a & 151924.7 & 210620.3 & $4 \times 3000$ & 21 & 2.1 \\
HCG 74b & 151924.4 & 205323.4 & $4 \times 3000$ & 21 & 2.1 \\
HCG 79a & 155911.4 & 204514.9 & $4 \times 3000$ & 65 & 2.9 \\
HCG 79b & 155912.6 & 204547.1 & $4 \times 3000$ & 79 & 2.9 \\
HCG 79c & 155910.9 & 204541.4 & $4 \times 3000$ & 79 & 2.9 \\
\hline
\end{tabular}

$7435 \mathrm{~km} \mathrm{~s}^{-1}$, cannot reject the possibility that this group is merely an artifact of a projection effect along the line of sight. de Carvalho et al. (1997) numbered up to 17 members, which show a bimodal redshift distribution with a separation of $\approx 500 \mathrm{~km} \mathrm{~s}^{-1}$.

The group contains two dominant galaxies named "a" and "b" in the Hickson nomenclature. According to $\mathrm{MdOH}$, member "a" is a double-nucleated, early-type (E1) galaxy, and "b" is a Sc spiral seen nearly edge-on. The two central objects have been confirmed on the DB-Asiago image by BB99. Component "a" has two compact companions designated "c" and "d". The northern one, "c", is a radio source. Infrared and radio emission are detected from the spiral galaxy (H93). H93 estimates a median galaxy separation of $49 h^{-1} \mathrm{kpc}$ ( $h$ is the dimensionless Hubble constant $\left.H_{0} /\left[100 \mathrm{~km} \mathrm{~s}^{-1} \mathrm{Mpc}^{-1}\right]\right)$ and a radial velocity dispersion of $209 \mathrm{~km} \mathrm{~s}^{-1}$.

Our contribution to the description is as follows. On the DB-Asiago and PH-CFHT images, we noticed that the outer isophotes of "a", although distorted by the presence of "d", are actually those of an E3 elliptical with a major axis at $\mathrm{PA}=23^{\circ}$. The unperturbed part of the isophotes have a center coinciding roughly with one of the central luminosity peaks (let us designate it by "n1"). The second nucleus ("n2") is significantly brighter; their separation is $4^{\prime \prime}$, and their relative position is at $\mathrm{PA}=135^{\circ}$.

With the aim of investigating the very nature of " $\mathrm{n} 2$ ", we have expanded the luminosity distribution of the $R$ band PH-CFHT frame into a series of Gaussian components grouped into three sets centered, respectively, on "a" (i.e., "n1"), "n2", and "d". We have followed a technique successfully applied to E and S0 galaxies (e.g., Emsellem et al. 1996; Loyer et al. 1998). The dimension, central brightness, axial ratio of individual components of each set, together with the central coordinates and position angle of these sets, where left as free parameters and were determined in a region of radius 40 " around "a". Starting with a reduced number of components, inspection of the residuals between the model and the observed distribution allowed us to add more components to each set, and thus to optimize the determination of the parameters. Typically, for early-type galaxies, the final rms residual is smaller than $10 \%$ in intensity. In the present case, we found the following:

- With a total of 15 components, the overall quality of fit is lower than expected: although a couple of percent in many areas, the rms residual is as high as $20 \%$ in others, distributed into a chaotic pattern with a cellular dimension of $\approx 10^{\prime \prime}$.

- Despite these limitations, a rather clear picture of " $\mathrm{n} 2$ " has emerged: it turned out to be the innermost region of an E3 galaxy with an effective radius of $\simeq 4.5^{\prime \prime}$; it is both larger and brighter than "d". Its absolute luminosity is $M_{B}=-21.0$ (for $h=0.75$ ), and its photometric profile (Fig. 2) does not appear significantly stripped off in the outer parts; we conclude that "n2" is unlikely to lie at the minimum distance to " $\mathrm{n} 1$ ", the nucleus of "a", allowed by the respective lines of sight, i.e., $4^{\prime \prime}$ or $\simeq 2 \mathrm{kpc}$.

- A very faint excess of light is seen close to "n2", as a long, flat structure likely too faint to be a galaxy of its own (its surface brightness is $\approx 1 \%$ of the central brightness of " $\mathrm{n} 2$ "); whether it is connected to " $\mathrm{n} 2$ ", thus suggesting an ongoing merger, or not, is unclear.

- The profile of "a" (Fig. 2, top panel) is similar to many other galaxies in compact groups (Bettoni \& Fasano 1993: hereafter BF93); these authors suspected such galaxies of harboring a tidal halo; more unexpectedly, the profile of "a" is also very similar to that of an archetypal cluster cD, NGC 6166 in Abel 2199 (Oemler 1976; Schombert 1986), which is twice as bright: this, together with the irregular luminosity distribution, and the presence of diffuse X-rays, is evidence for an evolution marked by mergers.

\subsubsection{Spectroscopy}

We have obtained kinematical data along a line at PA $=37^{\circ}$ cutting the "n1 $-\mathrm{n} 2$ " system roughly through the 


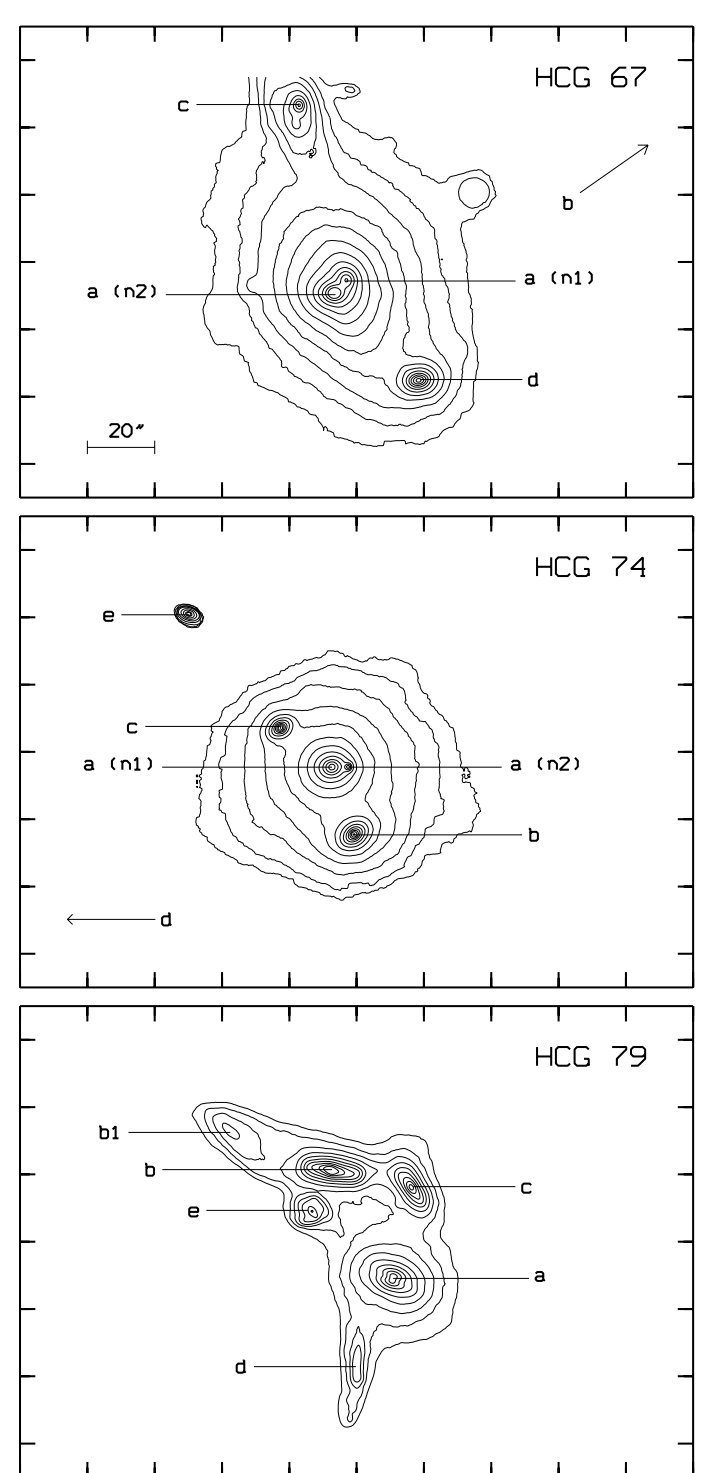

Fig. 1. Isophote maps from the $R$-band PH-CFHT frames. North is at the top, East to the left. The outermost isophotes correspond to $\mu_{R}=24.5,25.0$, and 23.5, respectively, for HCG 67, HCG 74, and HCG 79; the interval in magnitude is 0.5. Several stars and background galaxies have been removed; for HCG 67, the contour of the outermost isophotes is perturbed by an imperfectly removed bright star. The arrows indicate the approximate direction of a component out of the graph

apparent barycenter of the light distribution; the slit cuts "d" at about 4 " of its nucleus, and at an inclination of about $53^{\circ}$ with respect to its major axis. On this line, the brighter point in "a", which is the origin of our abscissae, coincides within one or two arcsec with the distance to "n2". We found (Fig. 3) the following:

- On the NE side, "a" exhibits a significant rotation, with an amplitude of $\simeq 200 \mathrm{~km} \mathrm{~s}^{-1}$, not inconsistent with the value expected for an E3 elliptical with a cen-
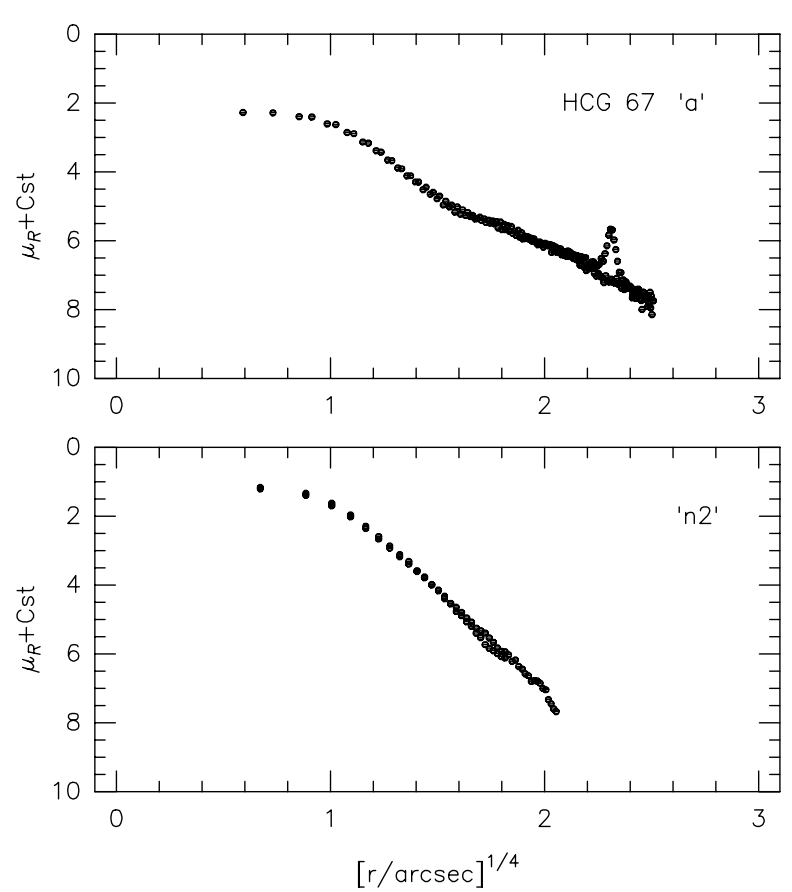

Fig. 2. HCG 67. $R$-band photometric profiles of components "a" (top panel) and "n2" (bottom panel), folded around the center. Both profiles are at $\mathrm{PA}=89^{\circ}$, which is the major axis of "n2". The ordinates, in magnitude scale, have been shifted by an arbitrary value, the same for "a" and "n2"

tral velocity dispersion of $291 \mathrm{~km} \mathrm{~s}^{-1}$ and isotropic dynamics.

- On the SW side, between $r \simeq 3$ and $15^{\prime \prime}$, we note a perturbation in the velocity and a rise of the dispersion up to $380 \mathrm{~km} \mathrm{~s}^{-1}$ at $r=7^{\prime \prime}$. This is in agreement with $\mathrm{BB} 99$, whose spectrum at $\mathrm{PA}=17^{\circ}$ shows the same behavior in a region located at a few arcsec from ours. There is another strong perturbation, between 15 " and $30^{\prime \prime}$ from "a", toward "d".

- Simple simulations involving template spectra show that kinematic perturbations similar to those observed can be generated by mixing the light from two objects with differential radial velocities of the order of 100 to $200 \mathrm{~km} \mathrm{~s}^{-1}$ (this was already noticed by Combes et al. 1995). $V$ shifted and $\sigma$ is increased, and when the difference in velocity is sufficient, the error bars on $V$ and $\sigma$ are also increased; this reflects the conflict between the actual, twin-peaked line-of-sight velocity distribution and the single Gaussian assumed in the "standard" reduction. This is likely to explain the observed features in the region of the optical transition between "a" and "d". Closer to "a", the maximum at $r=7^{\prime \prime}$ may also be due to the optical superposition of "n2", although, as an alternative, a physical effect like tidal heating cannot be ruled out.

BB99 have also obtained the gas kinematics of member "c" (Scd) of HCG 67, which has a rotation curve typical 

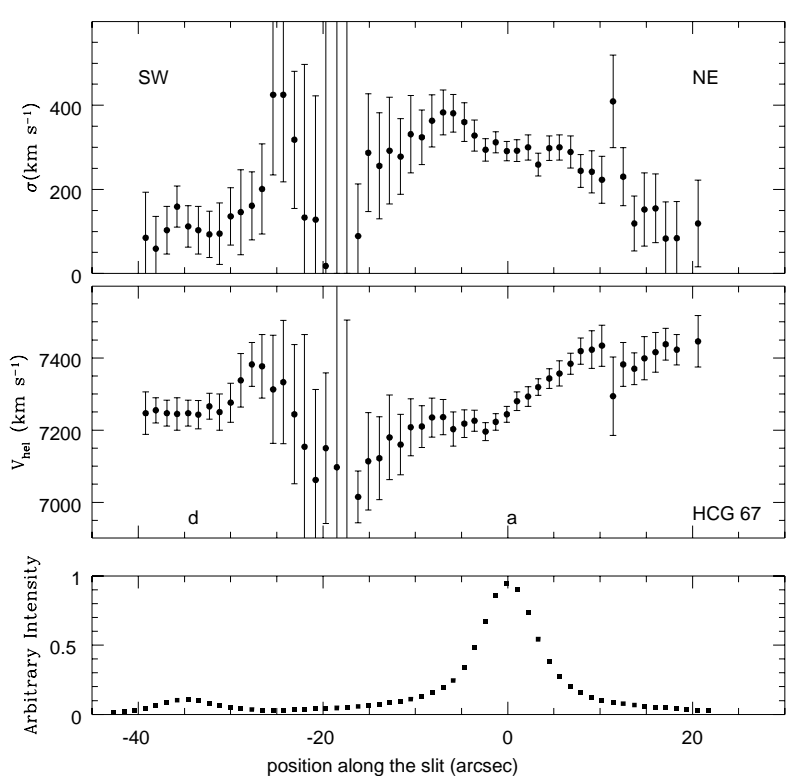

Fig. 3. HCG 67. Profiles of velocity dispersion $\sigma$ (top panel) and radial velocity $V_{\text {hel }}($ middle $)$ along a line at $\mathrm{PA}=37^{\circ}$ connecting the nuclei of the "a" and "d" members. Positions of the two components are indicated. Due to the smoothing of the spectrum in low-flux regions, individual points are not fully independent. Bottom panel: surface brightness $I$ along the same line, as measured on the spectrum

of a spiral, with a maximum velocity of $\approx 250 \mathrm{~km} \mathrm{~s}^{-1}$. The galaxy also exhibits an asymmetric structure, and a feature resembling a quite open arm which, given the high inclination of the equatorial plane, would be distorted; this may be indicative of an ongoing interaction (see Noguchi 1990).

\section{2. $H C G 74$}

\subsubsection{Morphology}

The group is a quintet composed of early-type galaxies; it is dominated by an elliptical galaxy, member "a", with two very close companions, "b" and "c". The elliptical is a radio source. All members have accordant redshift. H93 gives a median galaxy separation of $39 h^{-1} \mathrm{kpc}$, while the radial velocity dispersion is $316 \mathrm{~km} \mathrm{~s}^{-1}$.

Our image confirms the detection by $\mathrm{MdOH}$ and BF93 of a second nucleus (hereafter "n2") embedded in "a", at 5 " West of the main nucleus ("n1"). For this, on the $R$ band $\mathrm{PH}-\mathrm{CFHT}$ image, we have expanded the light distribution of "a" into a multi-Gaussian function, and we have subtracted this model from the observed frame. On the resulting image, flat to better than $1 \%$ in intensity, we have measured the profile of " $\mathrm{n} 2$ " $(1.10 \pm 0.05$ " FWHM), and found it slightly wider than stars $\left(0.85 \pm 0.10^{\prime \prime}\right.$ FWHM); thus, there is a fair level of confidence that it is not stellar. But on the map of the residuals, we did not see any
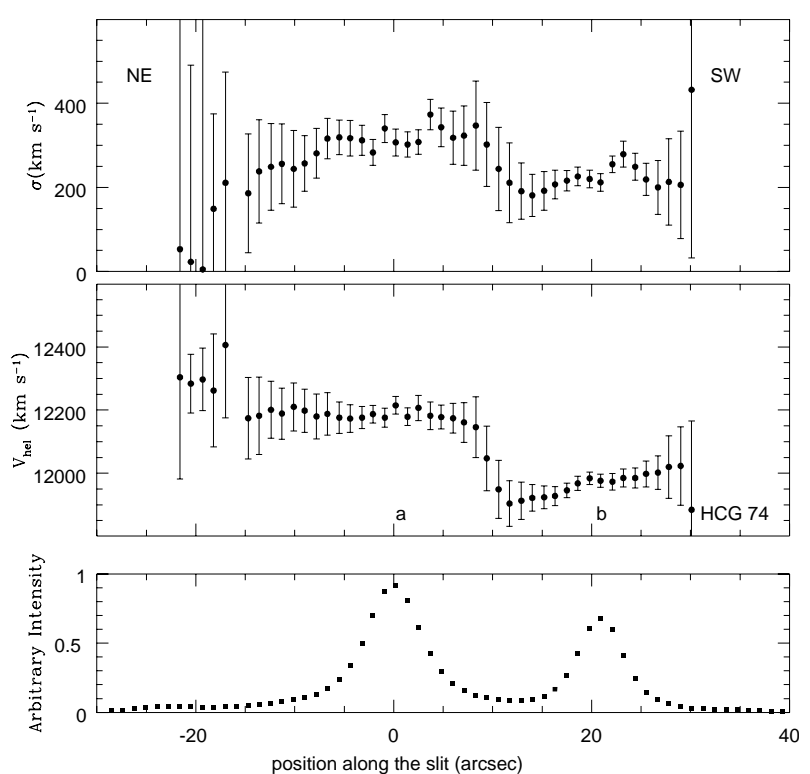

Fig. 4. HCG 74. Profiles of $\sigma, V_{\text {hel }}$, and $I$ along a line at $\mathrm{PA}=21^{\circ}$ connecting the nuclei of the "a" and "b". The faint outskirts of the component "e" are visible at $\approx 19^{\prime \prime}$ in the NE direction

pattern (at a contrast level of 1\%) that would reveal ripples or other evidence for a physical interaction, and the possibility remains that it is rather a foreground or background object. Because of the fairly circular isophotes of the model, we would classify "a" as E0 rather than E1.

\subsubsection{Spectroscopy}

Our kinematic measurements show a visible transition between "a" and "b" between $\simeq 8$ " and $\simeq 15$ " (see Fig. 4), with an increase of the velocity dispersion due to the mixing of the light from the two objects. The rotation of the two components is marginal, because the slit position is close to the minor axis for both; in addition, "a" is an E0 with no expected intrinsic rotation. For "b", a slightly concave shape for the inner rotation is due to the fact that the slit narrowly misses the nucleus.

\section{3. $H C G 79$}

\subsubsection{Morphology}

This group is known as Seyfert's Sextet and is the most compact one in Hickson's catalog, having a median intergalaxy separation of only $6.8 \mathrm{~h}^{-1} \mathrm{kpc}$. It has a relatively low radial velocity dispersion of $138 \mathrm{~km} \mathrm{~s}^{-1}$. H93 catalogs 5 members in this group, four of which have an accordant redshift. Member "e" (not reported in Table 2) has in fact a systemic velocity of $19809 \mathrm{~km} \mathrm{~s}^{-1}$. Inspection of the PH-CFHT images led to the following remarks. 
Table 2. Basic properties of member galaxies from the literature

\begin{tabular}{llrrl}
\hline $\begin{array}{l}\text { Member } \\
\text { ident. }\end{array}$ & Type & $\begin{array}{r}B_{\mathrm{T}} \\
(\mathrm{mag})\end{array}$ & $\begin{array}{r}V_{\text {hel }} \\
\left(\mathrm{km} \mathrm{s}^{-1}\right)\end{array}$ & Notes \\
\hline HCG 67a & E1 & 12.74 & 7262 & two nuclei - disturbed morphology \\
HCG 67b & Sc & 13.89 & 7644 & dust lane across the galaxy \\
HCG 67c & Scd & 15.06 & 7430 & asymmetric morphology, close companion \\
HCG 67d & S0 & 15.25 & 7071 & may have non concentric isophotes \\
& & & & \\
HCG 74a & E1 & 14.06 & 12255 & double nucleus, non concentric isophotes towards E, strong radio source \\
HCG 74b & E3 & 15.07 & 12110 & \\
HCG 74c & S0 & 16.10 & 12226 & \\
HCG 74d & E2 & 16.32 & 11681 & \\
HCG 74e & S0 & 17.80 & 11489 & \\
& & & & \\
HCG 79a & E0 & 14.35 & 4292 & Nuclear H $\alpha$ - strong dust lane - radio source \\
HCG 79b & S0 & 13.78 & 4446 & strong bar - tidal tail to the NE \\
HCG 79c & S0 & 14.72 & 4146 & extended envelope towards 79b \\
HCG 79d & Sdm & 15.87 & 4503 & asymmetric disk with a faint extension towards 79a \\
\hline
\end{tabular}

Notes. The values of the total $B$-mag and the systemic velocities of single members of the groups are obtained from Hickson (1993). Notes on morphological peculiarities are reported from Mendes de Oliveira \& Hickson (1994).
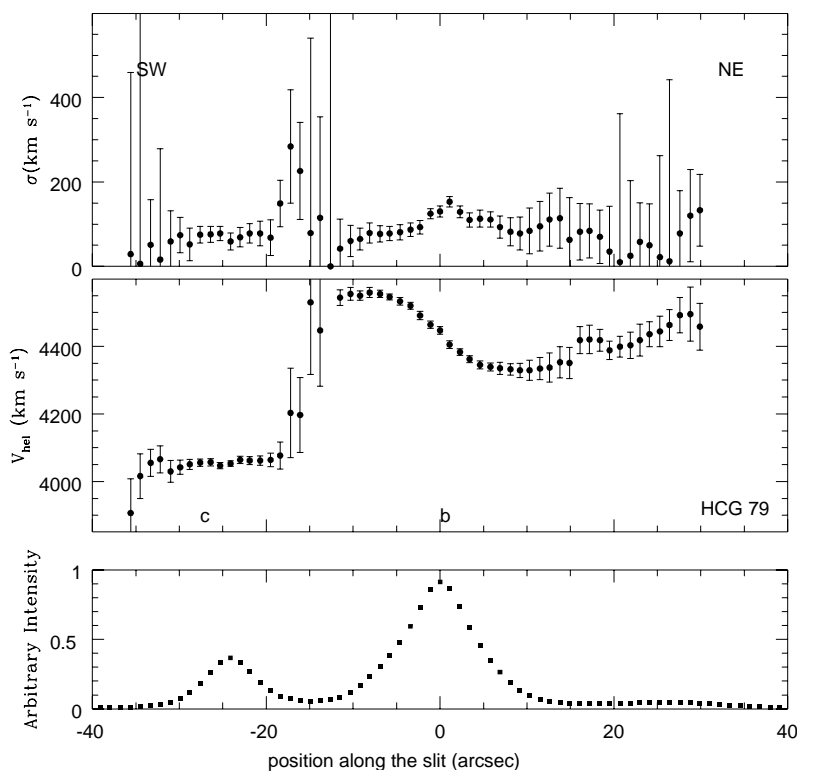

Fig. 5. HCG 79. Profiles of $\sigma, V_{\text {hel }}$, and $I$ along a line at PA $=$ $79^{\circ}$ connecting the nuclei of the "c" and "b"

The symmetric strong dust lane of "a" reveals a perfectly edge-on inclination; the inner isophotes are those of an E3 rather than an E0 elliptical, but it is embedded into an envelope which becomes gradually spherical outwards: this galaxy is thus similar in structure to a scaled-down M104.

Component "b" is clearly an edge-on S0 with an extended, flat disk. The object at $\approx 30^{\prime \prime}$ to the NE, designated by "b1" in BF93, is of uncertain nature. It could be a
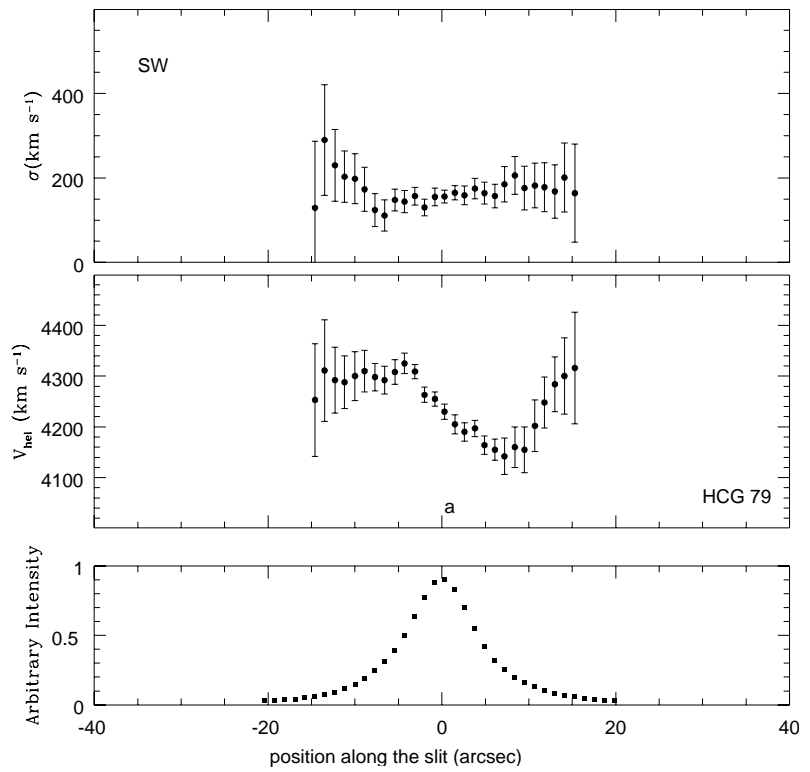

Fig. 6. HCG 79. Profiles of $\sigma, V_{\text {hel }}$, and $I$ along a line at PA $=$ $65^{\circ}$ connecting the nuclei of "a" and "b"

warped extension of "b" or, alternatively, another galaxy, so severely stripped off by tidal effects that it has lost most of its symmetric central condensation, and has adopted a comet-like appearance. Component "c" is also an edge-on S0, with a barely detected dust lane, and with slightly boxy outer isophotes; it is unclear whether the faint luminous bridge between "b" and "c" is anything more than the superposition of their individual brightnesses. 
Table 3. Main kinematic parameters from this study

\begin{tabular}{lrr}
\hline $\begin{array}{l}\text { Member } \\
\text { ident. }\end{array}$ & $\begin{array}{r}\mathrm{V}_{0, \text { hel }} \\
\left(\mathrm{km} \mathrm{s}^{-1}\right)\end{array}$ & $\begin{array}{r}\sigma_{0} \\
\left(\mathrm{~km} \mathrm{~s}^{-1}\right)\end{array}$ \\
\hline HCG 67a & $7246 \pm 24$ & $291 \pm 23$ \\
HCG 67d & $7247 \pm 36$ & $112 \pm 49$ \\
& & \\
HCG 74a & $12208 \pm 32$ & $312 \pm 31$ \\
HCG 74b & $11976 \pm 21$ & $212 \pm 21$ \\
& & \\
HCG 79a & $4237 \pm 18$ & $155 \pm 16$ \\
HCG 79b & $4447 \pm 11$ & $130 \pm 13$ \\
HCG 79c & $4053 \pm 08$ & $59 \pm 20$ \\
\hline
\end{tabular}

We also note the warped disk of the edge-on spiral "d" (already noted by $\mathrm{MdOH}$ ), which we regard as evidence of tidal interaction.

\subsubsection{Spectroscopy}

RFH give the rotation curve for "d", whose linear form is consistent with its edge-on orientation. A H I map obtained by Williams et al. (1989) shows the same range of velocities across the $\mathrm{H}$ I disk associated with the "d" galaxy as is seen in the RFH optical spectrum. These authors detect only weak nuclear emission for "a" and "b". RFH suggest that the components of HCG 79 are interacting also on the basis of another $\mathrm{H}$ I observation (Williams et al. 1989), which detected a tidal tail extending from "d" towards "a".

We succeeded in determining the velocity and velocity dispersion profiles of " $\mathrm{b}$ " up to the fainter "c" component visible at about $30^{\prime \prime}$ towards the $\mathrm{SW}$. We can make the following comments.

- In "b", at distances less than $\simeq 12$ ", the rotation and dispersion profiles are typical of a normal S0 galaxy observed almost edge-on. The velocity profile is very perturbed towards the SW just starting at $r=10^{\prime \prime}$. Whether this is due to the sole light mixing between "b" and "c" is still unclear.

- To the NE, the rotation of "b" falls to zero at $r \simeq 12$ ", and shows unambiguous counter-rotation beyond; this is likely due to the low-luminosity extension. It is unclear whether this is just the effect of an optical superposition, or is actually revealing the movement of some tidally perturbed material (from "b" itself or from the low-luminosity companion).

- Member "c" shows no rotation along the direction of the slit, which is at $\approx 45^{\circ}$ from its major axis. Since "c" is significantly flattened, this lack of rotation indicates that, either: i) it is oblate and inclined with respect to the line of sight, with an anisotropic dynamics, or ii) it is prolate, and it is rotating (or tumbling) around an axis coincident with the line of sight. Both cases would be, statistically, expected for a galaxy much more massive than this one $\left(M_{B}=-19.04\right)$. This off-average behavior could be regarded as a (mid) evidence of physical interaction within the group.

- The kinematics of component "a" has also been obtained along the direction $\mathrm{PA}=65^{\circ}$ (Fig. 6), which corresponds to the galaxy major axis (see also BF93). While the "c" and "b" members are S0 galaxies, "a" is classified as elliptical. The rotation curve seems nearly unperturbed in the SW side while the NE side, i.e., towards the rest of the group, shows a steep rise.

\section{Summary and discussion}

With the aim of studying the effect of the environment on the physics of early-type galaxies, we have started to collect relevant spectroscopic data, and this paper presents kinematical results for seven galaxies in the Hickson Compact Groups 67, 74, and 79. Stellar kinematics are seldom available for members of such groups, and our modest sample already represents a substantial fraction of the amount available in the literature. All the groups examined display very compact configurations in the sky, are mainly composed of early-type galaxies and, according to the literature, show several morphological signature of interaction. One of them, HCG 67 , is embedded withing an X-ray diffuse background while HCG 74 and HCG 79 have only an upper limit measured (Ponman et al. 1996).

We have looked for perturbations in the kinematical profiles, and we have carefully examined broad-band images in search of morphological peculiarities which could possibly be associated to these perturbations. In this task, we have attributed more weight to the shape of the rotation-velocity curve than to the velocity-dispersion one, as likely to be more unambiguously connected to gravitational interactions (Combes et al. 1995). Our measurements show several cases of apparent kinematical interactions, but for all of them, the mere optical superposition cannot be ruled out as the explanation.

Morphological evidence of physical interaction is sometimes elusive, and, for example, the reality of multiple nuclei also suffers from the ambiguity of the position along the same line of sight. For HCG 67 , we have been able to show that the putative second nucleus is actually another, smaller galaxy; there is no compelling evidence that it is undergoing a merging process. The fine structure of the central galaxy is, for this group, the strongest optical indication of a connection with the neighborhood. Convincing signature of severe gravitational perturbation is provided by warped disks and irregular extensions, but they cannot be considered as more than mild evidence of interaction within a physical group.

Rampazzo et al. (1998) noticed that morphological signature, kinematical features and the presence of hot gas 
are poorly correlated with one another. This also emerges from the present study. We consider the lack of compelling results in the present paper as an incentive to widen our sample and diversify our approach. We will gather spectroscopic and photometric data in compact systems, and also in looser ones, where superposition effects would be less severe. We plan to pay attention, again, to kinematical signatures if they appear unambiguous, but also to scaling relations which would link together several photometric and dynamical aspects. For this, a significant sample is needed, and we consider the present one as a first step.

Acknowledgements. We are deeply indebted to Daniela Bettoni for the permission to inspect her imaging material and kinematic data on HCG 67 before publication; we are also very grateful to Paul Hickson for the permission to use his CFHT images. We thank the referee, Giovanni Busarello, for his very careful and detailed comments on the initial version of the paper. We thank Gregory Maubon, and also the telescope operators of the Observatoire de Haute-Provence for their help in securing the observations.

\section{References}

Athanassoula E., Makino J., Bosma A., 1997, MNRAS 286, 825

Bettoni D., Buson L.M., 1999, in Small Galaxy Groups, IAU Colloquium No. 174, Flynn C., Valtonen M. (eds.), PASPC (in press) (BB99)

Bettoni D., Fasano G., 1993, AJ 105, 1291 (BF93)

Bonfanti P.P., Rampazzo R., Combes F., Prugniel Ph., Sulentic J.W., 1995, A\&A 297, 28

Combes F., Rampazzo R., Bonfanti P.P., Prugniel Ph., Sulentic J.W., 1995, A\&A 297, 37

Davoust E., Prugniel Ph., 1988, A\&A 201, 30

de Carvalho R.R., Ribeiro A.L.B., Capelato H.V., Zepf S.E., 1997, ApJS 110, 1

de Carvalho R.R., Coziol R., 1999, AJ 117, 1657 (dCC99)

dos Santos S., Mamon G.A., 1999 (in preparation)

Emsellem E., Bacon R., Monnet G., Poulain P., 1996, A\&A 312,777

Franx M., Illingworth G.D., Heckman T., 1989, ApJ 344, 613

Hernquist L., Katz N., Weinberg D., 1995, ApJ 442, 57

Hickson P., 1982, ApJ 255, 382

Hickson P., Mendes de Oliveira C., Huchra J.P., Palumbo G.G., 1992, ApJ 399, 353

Hickson P., 1993, ApL\&C 29, Nos. 1-3, 1

Huchtmeier W.K., 1997, A\&A 325, 473

Leon S., Combes F., Menon T.K., 1998, A\&A 330, 37

Longo G., Busarello G., Lorenz H., Richter G., Zaggia S., 1994 ,
A\&A 282, 418

Loyer E., Simien F., Michard R., Prugniel Ph., 1998, A\&A 334, 805

Mamon G.A., 1986, ApJ 307, 426

Mamon G.A., 1987, ApJ 321, 422

Mamon G.A., 1992, ApJ 401, L3

Mamon G.A., 1994, in Cluster of Galaxies, Durret F., Mazure A., Tran Thanh Van J. (eds.). Éditions Frontières, Paris, p. 297

Mendes de Oliveira C., Hickson P., 1994, ApJ 427, 684 (MdOH)

Mendes de Oliveira C., Plana H., Amram Ph., Bolte M., Boulesteix J., 1998, ApJ 507, 691

Mendes de Oliveira C., Amram Ph., Balkowski Ch., Boulesteix J., 1997, in Dark and visible matter in galaxies, Persic M., Salucci P. (eds.) PASPC 117, 156

Menon T.K., 1995a, MNRAS 274, 845

Menon T.K., 1995b, AJ 110, 2605

Moles M., del Olmo A., Perea J., Masegosa J., Marquez I., Costa V., 1994, A\&A 285, 404

Oemler A., 1976, ApJ 109, 693

Ostriker J.P., Lubin L.M., Hernquist L., 1995, ApJ 444, L61

Noguchi M., 1990, in Dynamics and Interaction of Galaxies, Wielen R. (ed.). Springer-Verlag, Berlin, Heidelberg, p. 469

Pildis R.A., 1995, ApJ 455, 492

Plana H., Mendes de Oliveira C., Amram Ph., Boulesteix J., 1998, AJ 116, 2123

Ponman T.J., Bourner P.D.J., Ebeling H., Böhringer H., 1996, MNRAS 283, 690

Rampazzo R., Covino S., Trinchieri G., Reduzzi L., 1998, A\&A 330,423

Ribeiro A.L., de Carvalho R.R., Capelato H.V., Zepf S.E., 1998, ApJ 497, 72

Rubin V.C., Ford W.K., Hunter D.A., 1991, ApJS 76, 153

Schombert J.M., 1986, ApJS 60, 603

Simien F., Prugniel Ph., 1997a, A\&AS 122, 521

Simien F., Prugniel Ph., 1997b, A\&AS 126, 15

Simien F., Prugniel Ph., 1997c, A\&AS 126, 519

Simien F., Prugniel Ph., 1998, A\&AS 131, 287

Sulentic J.W., de Mello Rabaça D.F., 1993, ApJ 410, 520

Sulentic J.W., Rabaça C.R., 1994, ApJ 429, 531

Venugopal V.R., 1995, MNRAS 277, 455

Verdes-Montenegro L., del Olmo A., Perea J., Athanassoula E., Marquez I., Augarde R., 1997, A\&A 321, 409

Verdes-Montenegro L., Yun M.S., Perea J., del Olmo A., Ho P.T.P., 1998, ApJ 497, 89

White S.D.M., Huchra J., Latham D., Davis M., 1990, in Dynamics and Interaction of Galaxies, Wielen R. (ed.). Springer, Heidelberg, p. 380

Williams B.A., Rood H.J., 1987, ApJS 63, 265

Williams T.B., Schommer R.A., Bothun G.D., Mould J.R., 1989, BAAS 21, 1128

Zepf S.E., 1993, ApJ 407, 448 